\title{
Foro de Análisis Financiamiento e Inclusión Financiera para el Desarrollo Agropecuario, Rural y de Sistemas Alimentarios en América Latina y el Caribe: Documento Resumen
}

Eugenio Díaz-Bonilla, Eduardo Fernández-Arias, y Valeria Piñeiro, con Bettina Prato y Joaquín Arias. 


\section{CONTENTS}

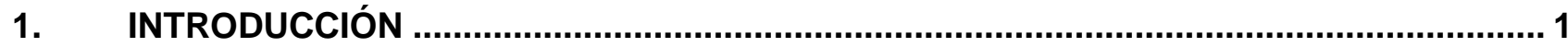

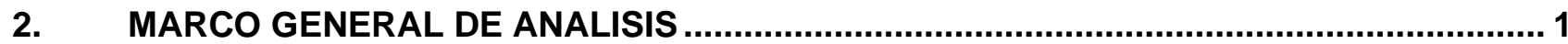

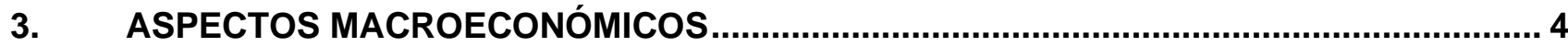

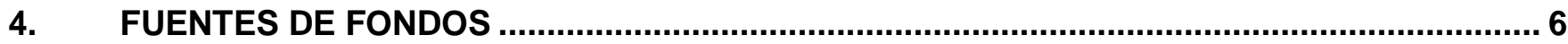

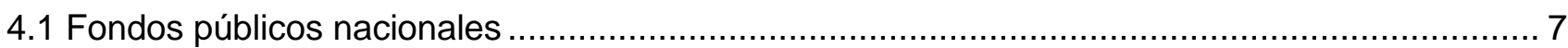

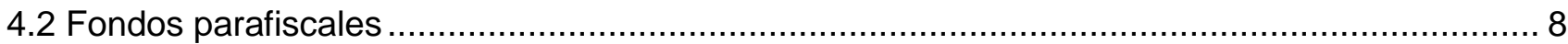

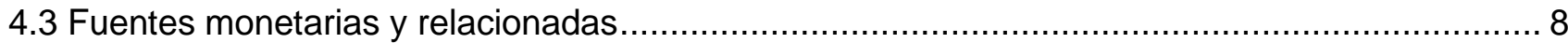

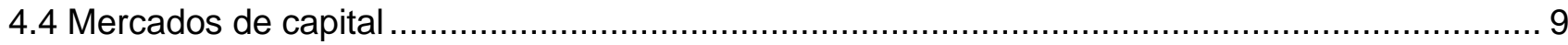

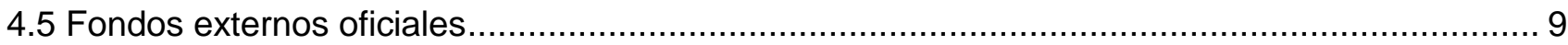

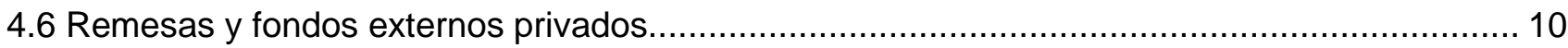

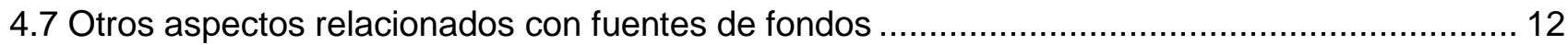

5. INSTITUCIONES FINANCIERAS Y REGULACIONES $\ldots$

5.1 Bancos públicos y privados. Primero y segundo piso ............................................................. 13

5.2 Cooperativas, bancos comunitarios y microcrédito.............................................................. 14

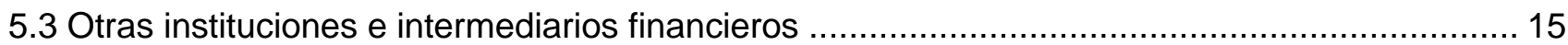

6. MANEJO DEL RIESGO EN LAS ENTIDADES FINANCIERAS...............................................

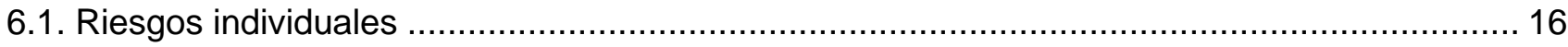

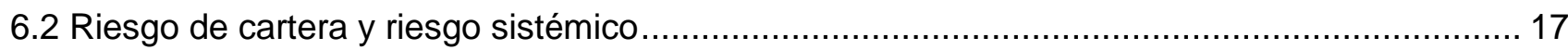

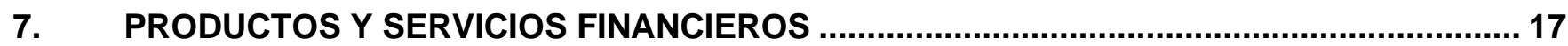

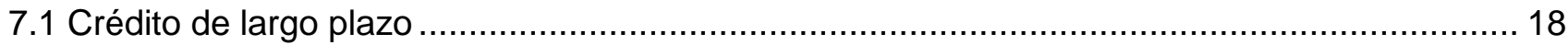

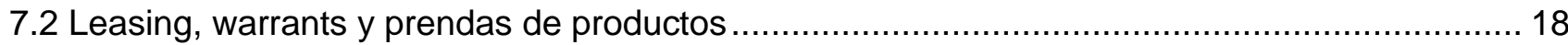

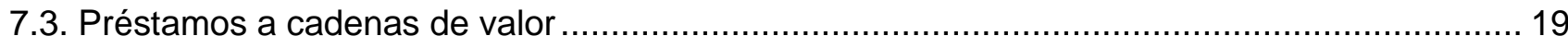

7.4. Garantías, pagos flexibles y préstamos con insumos ......................................................... 19

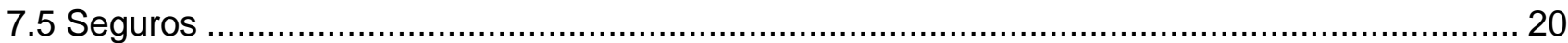

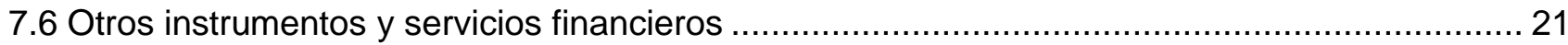

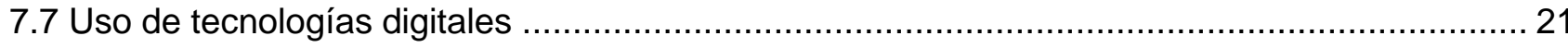

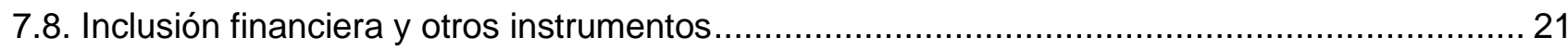

8. PRODUCTOS Y SERVICIOS DE APOYO AL FINANCIAMIENTO …........................................ 22 


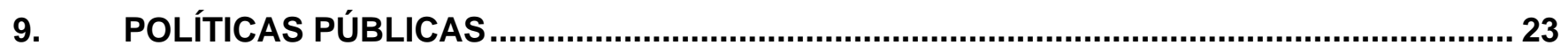

10. SIGUIENTES PASOS: POSIBLES AGENDAS DE TRABAJO CONJUNTO ............................ 25

ANEXO

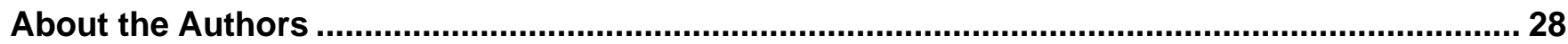

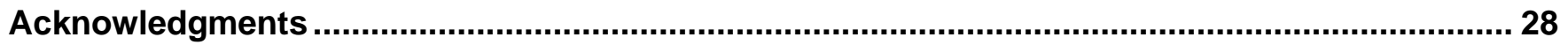

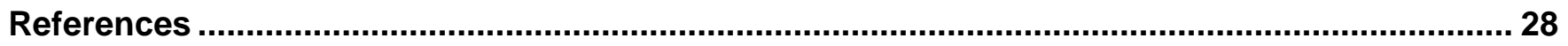




\section{INTRODUCCIÓN}

IFPRI e IICA, con el apoyo del Secretariado de la Red para la Inversión y la Financiación de la Agricultura en Pequeña Escala (SAFIN en inglés) con sede en FIDA, organizaron el 6 y 7 de mayo de 2019 en la sede del IICA en San José, Costa Rica, un foro para analizar la experiencia en materia de financiamiento e inclusión financiera para el desarrollo agropecuario, rural y de sistemas alimentarios en América Latina y el Caribe (ALC). El objetivo ha sido construir una agenda de temas a analizar para derivar propuestas apropiadas de política económica en esas áreas. Previamente se distribuyó también un documento base para la discusión (Díaz-Bonilla y Fernández-Arias, 2019). Se invitó a especialistas de diferentes países, representando a organismos públicos, al sector privado, incluyendo grupos de productores agropecuarios, sociedad civil, organismos internacionales y organizaciones financieras de desarrollo no gubernamentales.

El evento se inició con una presentación del Director General del IICA sobre la situación general del sector agropecuario en la región. Las sesiones temáticas se organizaron con la siguiente estructura: a) una presentación general para dar un marco a la discusión (usando especialmente el documento base mencionado, Díaz-Bonilla y Fernández-Arias, 2019); b) la propuesta de diferentes preguntas indicativas para el análisis; y 3) las intervenciones de los participantes.

A continuación, se resumen diferentes aspectos del rico debate y análisis durante el foro, siguiendo básicamente la estructura de temas propuestos en la agenda.

\section{MARCO GENERAL DE ANALISIS}

Un tema inicial fue el alcance de la discusión del foro. Por ejemplo, se preguntó si el análisis debía centrarse solamente en las instituciones bancarias públicas, o extenderse a otras instituciones financieras privadas. También se mencionó si la discusión era sobre financiamiento agropecuario, de manera más acotada, de cadenas de valor (con una visión por productos), de desarrollo rural (con una visión territorial), o si se debía tomar una perspectiva más general considerando la necesidad de financiamiento para la transformación de los sistemas agro-alimentarios (lo que involucra romper en parte la dicotomía rural-urbana). En general, se consideró tomar una visión amplia de los temas involucrados. 
Esto estaba relacionado con la pregunta de fondo sobre por qué era necesario (o no) analizar nuevamente el papel de los sistemas bancarios/financieros en América Latina y el Caribe (ALC) y de las políticas públicas e instituciones relacionadas en este nuevo contexto, en lo que hace al desarrollo agropecuario, rural y de los sistemas agro-alimentarios en la región. ${ }^{1}$

En la presentación marco hubo una reseña del debate acerca de los sistemas bancarios/financieros en países en desarrollo, y su papel específico en relación con el sector agropecuario (ver también DíazBonilla y Fernández-Arias, 2019). El enfoque tradicional fue intervencionista con topes a las tasas de interés de los préstamos, regulaciones para dirigir el crédito privado, y crédito subsidiado a través de bancos públicos. En el caso del sector agropecuario ese crédito se orientó el financiamiento hacia productos prioritarios y/o a fomentar la adopción de ciertas tecnologías más modernas. También se veía al financiamiento como un medio para ayudar a pequeños productores a salir de la pobreza.

Este enfoque (que algunos llamaron de "represión financiera") fue criticado porque llevó a consecuencias no deseadas, tales como el racionamiento regresivo y distorsiones ineficientes del crédito privado; una banca privada débil sin incentivos para el desarrollo financiero para el ahorro; alto costo fiscal de bancos públicos por captura y mal manejo; y la presencia de prácticas corruptas. Por ende, se pasó a enfatizar el fortalecimiento del sistema bancario/financiero para que pudiera cumplir sus funciones básicas en general y en el ámbito rural especialmente: a) intermediar adecuadamente el ahorro y la inversión; b) servir de mecanismo de pagos; y c) ayudar a manejar el riesgo de los agentes económicos. La idea era que, si los sistemas bancarios/financieros cumplían adecuadamente sus funciones básicas, no hacían falta políticas públicas que los forzaran a atender otros objetivos.

En el caso de la banca pública agropecuaria, frente la opción que fue caracterizada como de "Cierre" o "Reforma" durante los años 80 y 90, ALC se inclinó más por la liberalización del sistema financiero con cierre de la banca de desarrollo (lo que también fue el caso de Africa), mientras que muchos países de Asia, por ejemplo, tomaron la opción de reforma y reestructuración de la banca de desarrollo. ${ }^{2}$

\footnotetext{
${ }^{1}$ En el documento base se mencionaban una serie de trabajos previos relacionados, tales como Adams, Graham, y von Pischke, ed. 1984; FAO y GTZ "Agricultural Finance Revisited" (que produjeron una serie de trabajo a fines de los 1990s). Por la misma época, IFPRI realizó un amplio estudio multipaís sobre microfinanzas (Manfred Zeller y Richard Meyer eds. 2002). En ALC y más recientemente, están los estudios de Trivelli y Venero (2007), trabajos del BID en la mitad de la década de los años 2000, y diferentes estudios de IICA (cadenas de valor). Desde la crisis económica y financiera global, el G20 dio lugar a la Asociación Global para la Inclusión Financiera (Global Partnership for Financial Inclusion, GPFI), que tiene una línea de trabajo de apoyo a las pequeñas y medianas empresas (PyMEs), incluyendo las agropecuarias y otros aspectos de inclusión financiera en áreas rurales. Por su parte el FIDA y otras instituciones crearon en 2017 la Red para la Inversión y la Financiación de la Agricultura en Pequeña Escala (SAFIN, por sus siglas en inglés).

${ }^{2}$ En el documento base se cita el trabajo de Seibel, Giehler y Karduck (2005) que argumenta con ejemplos que es posible la reforma exitosa en base a una intermediación financiera rentable. Fernández-Arias, Hausmann y Panizza (2019) examinan el rediseño de la banca de desarrollo para la época actual.
} 
ALC fue la región que más liberalizó su sistema financiero, pero eso no se reflejó en un aumento similar del crédito (ver Díaz-Bonilla y Fernández-Arias, 2019 y Anexo con cuadros presentados durante el foro).

La pregunta es entonces si es tiempo de retomar el análisis de estos temas para ALC incorporando las experiencias más recientes y con una perspectiva integradora. La hipótesis de este evento fue que parecía necesario reexaminar la experiencia de la región en lo que hace a los mercados financieros rurales, bajo las nuevas circunstancias de desarrollo financiero, tecnológicas y globales., Esto incluye la necesidad de adecuar los sistemas agro-alimentarios para cumplir los Objetivos de Desarrollo Sostenible (ODS) en el 2030, especialmente, ODS1 (pobreza); ODS2 (seguridad alimentaria y productividad); ODS3 (salud); ODS6 (agua); ODS7 (energía); ODS8 (crecimiento económico); ODS9 (infraestructura resiliente, e industrialización inclusiva); ODS10 (desigualdad); ODS 12 (consumo y producción sostenibles); ODS 13 (cambio climático); y ODS 15 (ecosistemas, tierras, biodiversidad).

Por ejemplo, en el foro se mencionaron los desafíos del cambio climático, el stress hídrico, y otros aspectos ecológicos relacionados, que pueden requerir importantes reconversiones de los sistemas productivos en la región. También se comentaron las implicaciones de los nuevos patrones de nutrición para atender diferentes problemas de malnutrición, incluyendo problemas de obesidad.. Si la demanda se reorientara de acuerdo con esas preocupaciones nutricionales, podría haber exceso de oferta de unos productos (por ejemplo, azúcar, palma africana, carne roja) y falta de otros (frutas, hortalizas, legumbres, nueces). Reestructurar el aparato productivo puede tener impactos importantes sobre productores y trabajadores rurales. ${ }^{3}$

Esta lleva a dos preguntas básicas: ¿Cuánto costaría la restructuración del sistema agro-alimentario para alcanzar esos objetivos? ¿Cómo se pueden financiar los gastos e inversiones para esa transformación?

El posible financiamiento incluye varias posibles fuentes (ver, por ejemplo, Schmidt-Traub y Sachs 2015; Díaz-Bonilla, 2018; Díaz-Bonilla y Callaway, 2018), pero una importante son los sistemas bancarios y financieros nacionales. Entonces, una discusión parte de esa discusión más general tiene que ver con el papel de los sistemas bancarios/financieros en el financiamiento de la reestructuración necesaria del sector agropecuario, rural y los sistemas agroalimentarios para alcanzar los ODS en 2030.

Adicionalmente, está el interés por ciertos objetivos societales que los sistemas bancarios/financieros podrían o deberían considerar, pero que por una variedad de fallas de mercado y/o de problemas de

\footnotetext{
${ }^{3}$ En la presentación marco para esta sesión se mencionó, por ejemplo, que azúcar representa alrededor del $11 \%$ del valor de la producción agropecuaria en ALC y las carnes rojas, el $20 \%$ ). Más en general, si hay que restructurar todo el sistema agroalimentario, y no solamente la producción primaria, esto tiene repercusiones sobre toda la economía: por ejemplo, el sistema agro-alimentario completo en Honduras representa el $29 \%$ del PBI y $40 \%$ del empleo; en Costa Rica, es $20 \%$ del PBI y $26 \%$ del empleo.
} 
equidad no estarían siendo atendidos. Por ejemplo, la inclusión financiera de pequeños productores y grupos vulnerables o ignorados (mujeres, etnias, jóvenes).

En todos esos casos la pregunta sería qué tipos de políticas públicas y reformas institucionales se necesitan para que los sistemas bancarios/financieros puedan generar inclusión financiera, y/o apoyar la transformación de los sistemas agro-alimentarios en América Latina y el Caribe (ALC) de manera de cumplir con los ODS relacionados con esos temas.

En general, y dada la variedad de intereses y enfoques representados por los participantes, se decidió adoptar una visión amplia para la discusión de problemas, objetivos, políticas publicas e instituciones. Esa discusión siguió la estructura siguiente: 1) aspectos macroeconómicos; 2) fuentes de fondos; 3) instituciones financieras, regulaciones y manejo del riesgo; 4) productos y servicios financieros; y 5) productos y servicios de apoyo. Se concluyó con una visión general sobre los criterios de políticas públicas en el sector. Como se mencionó cada sesión empezó con una presentación de marco y la propuesta de preguntas para discusión.

\section{ASPECTOS MACROECONÓMIICOS}

Un aspecto básico es el entorno macroeconómico (inflación, expectativas de devaluación, déficit fiscal, deuda externa, ciertas regulaciones, etc.) que afecta tanto la operación del sistema bancario/financiero, como el funcionamiento del sector agropecuario, de las economías rurales y de los sistemas agro-alimentarios (es decir la causalidad va del entorno macro hacia los otros sectores). A su vez, los sistemas bancarios/financieros y el sector agro-alimentario tienen implicaciones para la macroeconomía en general (en este caso es la causalidad inversa, de esos sectores hacia la macroeconomía).

A diferencia del período de bonanza previo, la coyuntura actual de América Latina y el Caribe, con la desaceleración del crecimiento, restricciones fiscales, y altos niveles de endeudamiento público, plantea un escenario distinto para el fortalecimiento de los sistemas financieros rurales.

Luego de la presentación general se plantearon las siguientes preguntas:

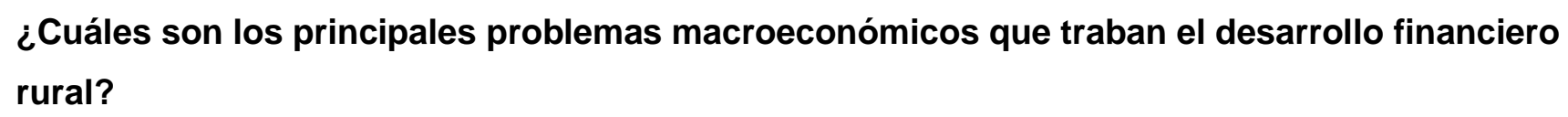
rural?

¿Hay regulaciones macro-prudenciales que sean particularmente negativas (o positivas) para el funcionamiento de los mercados financieros rurales y de las instituciones financieras que operan allí limitando la consecución de objetivos societales como por ejemplo los ODS? 
En relación con la primera línea de causalidad (de la macroeconomía hacia el sistema bancario/financiero, y los sistemas agro-alimentarios), se mencionó, por ejemplo, que con inflación de 40-50\% y tasas de interés de 60-70\%, como está sucediendo en la Argentina, el sistema bancario/financiero no puede cumplir sus funciones básicas en general, y no solamente en relación con el sector agro-alimentario.

Afortunadamente, el marco macroeconómico en muchos países de la región ha mejorado en las últimas décadas (aunque ha habido un deterioro últimamente) lo que facilita tanto la operación de los mercados financieros, como los aspectos productivos y por tanto, no hubo mayor discusión de estos temas. Sin embargo, la solvencia fiscal (en los casos en que el fondeo del crédito que provenga del presupuesto público) y el programa monetario (si el fondeo fuera a través de los bancos centrales) requerirían un análisis más detallado. En general, es importante la coherencia entre las políticas monetarias, fiscales y comerciales, ya que a veces las mismas actúan en contraposición empeorando el ambiente económico y financiero para los productores.

Hubo una referencia puntual al tema del esquema monetario y el tipo de cambio cuando se mencionó que Ecuador utiliza impuestos de importación o tasas aduaneras para compensar devaluaciones de países vecinos. Al estar dolarizado, este país no puede devaluar para absorber choques externos. Obviamente, el tipo de cambio, y su volatilidad, aunque no fueron mencionados, son importantes para la rentabilidad del sector agropecuario, en tanto se trata mayormente de productos comercializables internacionalmente (como exportables o como competidores de importaciones). Aún más, considerando que en varios países el sistema bancario muestra tanto depósitos (o fondeo en general) como préstamos y otras inversiones en dólares, el tipo de cambio y su variabilidad afectan directamente los activos y pasivos del sistema bancario, con riesgos de quebrantos tanto de los deudores, como de las entidades financieras.

Un tema relacionado a los aspectos macroeconómicos que sí motivó discusiones fue la volatilidad de precios agropecuarios y sus implicaciones para el sector productivo (lo que a su vez afecta el atractivo del sector agropecuario como sujeto de crédito para la banca). Se mencionaron la posibilidad de constituir un fondo de estabilización de precios en Colombia y el uso del mecanismo de franjas de precios en Ecuador para contrarrestar el impacto de lo que se consideran son precios distorsionados en los mercados internacionales. Ambas intervenciones tienen importantes limitaciones operacionales y en relación con las reglas de la Organización Mundial del Comercio (ver Díaz Bonilla 2017a and 2017b, sobre los aspectos operacionales y legales de los fondos de estabilización).

También se discutió la volatilidad de los precios de las "commodities" en los mercados internacionales, y en particular se mencionó el café. Se argumentó que la volatilidad de precios afectaba seriamente la 
rentabilidad de los productores de café y que eso estaba afectando su capacidad de repago a las instituciones prestatarias (incluyendo el caso de algunas entidades financieras internacionales de promoción social representadas en este evento y que están apoyando pequeños productores en esa cadena de valor). Hubo comentarios sobre la necesidad de estabilizar los precios internacionales y otras voces que manifestaron escepticismo sobre que eso pudiera hacerse. En todo caso, los gobiernos y entidades financieras deberían monitorear estos ciclos, y asesorar adecuadamente a los productores en sus decisiones productivas para evitar expansiones excesivas de cultivos permanentes durante períodos de precios altos que llevan luego a caídas de precios. También, considerando que esa volatilidad de precios es muy difícil de eliminar más bien lo que se requiere son seguros de precios o pensar en instrumentos de crédito que la tomen en cuenta con mecanismos de pago más flexibles, sobre todo considerando que la entidad financiera no elimina el riesgo simplemente por tener calendarios de pago rígidos, sino que simplemente lo transforma en la posibilidad de insolvencia del productor (ver más adelante).

Además de la línea de causalidad que va de la macroeconomía al sistema financiero y a la producción, también se mencionó en la presentación marco de la sesión la causalidad inversa: los mercados financieros rurales pueden ser relevantes para el riesgo de crisis bancarias y sistémicas, y por lo tanto los países utilizan políticas macro-prudenciales, por la potencial inestabilidad agregada (riesgos covariantes) de la economía rural.

En principio las regulaciones son para asegurar un funcionamiento adecuado del sistema bancario/financiero en las tres dimensiones mencionadas ${ }^{4}$, para manejar riesgos (crédito, liquidez, exposición cambiaria, fluctuaciones cíclicas) y evitar crisis. Ejemplos de regulaciones macroprudenciales incluyen coeficientes de adecuación de capital, coeficientes de liquidez, herramientas regulatorias contracíclicas, exposición cambiaria, topes de préstamos.

Al mismo tiempo, también se mencionó que es la regulación para mitigar riesgos puede afectar el desarrollo financiero rural y los objetivos de alivio a la pobreza, seguridad alimentaria, sostenibilidad ambiental e inclusión financiera (ver más adelante).

\section{FUENTES DE FONDOS}

Un tema importante a nivel macroeconómico es el flujo de fondos, es decir la matriz que muestra el origen de los ahorros (fuentes de fondos) y su asignación a diferentes usos o inversiones. Esas fuentes

\footnotetext{
${ }^{4}$ Como se mencionó las tres funciones fundamentales del sistema bancario son: a) como sistema de pago para la economía; b) como intermediario entre ahorros e inversiones y c) como proveedor clave de servicios de gestión de riesgos.
} 
de fondos pueden incluir, entre otros (1) el presupuesto público; (2) autoridades monetarias (redescuentos, reducción mínimos de reservas, préstamos en porcentaje de depósitos); (3) financiamiento del sector privado interno (autofinanciamiento, o ahorros de otros intermediados por el sistema bancario o por el mercado de capitales doméstico); y (4) financiamiento del resto del mundo (préstamos de organismos internacionales, entidades internacionales no gubernamentales de desarrollo, mercado de capitales externos).

En la presentación marco de esta sesión se mencionó que las fuentes de fondos del financiamiento rural han cambiado sustancialmente y sin mayor análisis en los últimos 20 años. Cada fuente tiene su propia lógica, y elementos a favor y en contra que conviene analizar. Por ello se planteó la siguiente pregunta:

\section{¿Cuáles son las diferentes maneras en que se fondean los bancos y entidades financieras que operan con el sector agropecuario, rural y los sistemas agroalimentarios? ¿Cuáles son los as- pectos positivos y negativos de cada una de ellas?}

\subsection{Fondos públicos nacionales}

El fondeo del presupuesto público puede ser una asignación de capital de una vez como un fideicomiso, o ser repetido varias veces cuando se necesita recapitalizar instituciones. En el caso del BNDES de Brasil una parte de los impuestos que se recaudan sobre los salarios de los trabajadores van al banco. Los fondos públicos pueden ser más útiles para financiamiento de largo plazo, pero pueden generar problemas fiscales si los préstamos no son repagados, y se requieren salvatajes permanentes de los bancos públicos (o privados, si los fondos fueran canalizados por instituciones privadas). Por otra parte, requerimientos muy estrictos de conservación del aporte de capital público pueden limitar el impacto de desarrollo si debido a esos requerimientos se manejan esos recursos con criterios comerciales cuando los objetivos de política económica pueden requerir, según algunas opiniones, un elemento de subsidio. Varios participantes mencionaron el peligro para los funcionarios públicos de sanciones administrativas y penales si hubiera pérdidas importantes, lo que lleva a criterios más conservadores en el uso de los recursos.

Un aspecto relacionado es el uso de garantías públicas para que bancos privados utilicen sus propios recursos en las actividades priorizadas por el gobierno. Hubo diferentes debates acerca de las diferentes regulaciones que limitan un uso más extendido de esas garantías por parte de los bancos e instituciones financieras privadas. 
También se discutió la posible tendencia a un mayor uso de fondos públicos en otros instrumentos para reducir el riesgo privado, tales como lo que se llama "blended finance."

\subsection{Fondos parafiscales}

Se analizó asimismo el tema de los fondos parafiscales ${ }^{5}$, como es el caso de Colombia, que financian diferentes actividades relacionadas con los productores, aunque no parecen incluir el crédito (o la provisión de garantías), y más bien se orientan hacia investigación agropecuaria extensión, extensión, y desarrollo de infraestructura.

\subsection{Fuentes monetarias y relacionadas}

En lo que hace a los aspectos monetarios, históricamente los bancos centrales dieron líneas de crédito para actividades prioritarias como el sector agropecuario, que podían o no estar coordinadas con el programa monetario general, y por tanto podían ampliar excesivamente la oferta monetaria y generar inflación. Algunos países de la región, como parece ser el caso de Honduras, tienen algún fondeo del banco central para actividades de desarrollo.

Un aspecto relacionado, porque también incrementa la oferta monetaria amplia con una asignación específica del flujo adicional, es el uso de encajes diferenciales para las actividades priorizadas (es decir, se establecen encajes menores cuando se trata de préstamos a actividades agropecuarias u otras que se quieren fomentar).

Un mecanismo de fondeo común basado en la regulación bancaria es la priorización selectiva de destinos de los depósitos de los bancos. Pero hay variaciones en lo que hace a la obligación o no de usar depósitos para financiar al sector agropecuario y actividades relacionadas. Colombia, por ejemplo, tiene un sistema por el cual una parte de los depósitos tiene que ser asignada al sector agropecuario. Pero los bancos, si no quieren prestar directamente ese porcentaje, tienen la opción de comprar Títulos de Desarrollo Agropecuario que a su vez se asignan a un fidecomiso que financia actividades productivas agropecuarias.

Mandatos de prestar cierto porcentaje de los depósitos (o de compras compulsivas de instrumentos financieros públicos) pueden, por una parte, ampliar la oferta de crédito para el sector; pero también

\footnotetext{
${ }^{5}$ Recursos que se recaudan con contribuciones similares a impuestos de los propios productores.
} 
pueden elevar el riesgo de los bancos y, si implican tasas de interés inferiores a las que esas entidades financieras pueden obtener en otras actividades, pueden reducir los ingresos y/o subir el costo de otras operaciones de los bancos.

Algunos países tienen depósitos en moneda local y moneda extranjera (dólares) como es el caso de Uruguay y Argentina, por ejemplo, lo que complica la operatoria crediticia en relación con quién asume el riesgo cambiario. Para evitar las crisis del pasado en las que fondos en dólares eran usados para financiar préstamos en moneda local, se han implementado regulaciones prudenciales que limitan el descalce de moneda; sin embargo, si el prestatario tiene sus ingresos en moneda local, el banco ha descargado el riesgo cambiario, pero ha asumido el riesgo de bancarrota del cliente en caso de una devaluación importante. Se mencionó en el foro que el Banco de la República Oriental del Uruguay maneja el descalce de moneda en parte invirtiendo los recursos de los depósitos en dólares en bonos del tesoro de los Estados Unidos. Eso asegura que la entidad no va a tener problemas de descalce de moneda (o peor, de corridas contra los depósitos en moneda extranjera), pero limita su capacidad de fomentar el desarrollo interno.

\subsection{Mercados de capital}

Algunos intermediarios financieros de segundo piso como el FIRA de México emiten títulos de deuda y bonos (incluyendo, por ejemplo, bonos verdes) en los mercados de capital locales e internacionales. Esa institución también ha realizado operaciones de titularización, en las que diferentes préstamos se colocan en un mismo paquete que luego se coloca en los mercados de capital domésticos con garantía de FIRA.

Se mencionó que los bancos privados también pueden financiarse en los mercados de capital locales o internacionales, pero que ese no es necesariamente el caso de intermediarios financieros especializados (como por ejemplo entidades de "leasing") que usualmente no tienen acceso a los mercados de capital y por tanto deben financiarse con bancos locales. Esto eleva el costo de los fondos y limita el desarrollo de ese tipo de instrumentos.

\subsection{Fondos externos oficiales}

Pasando a los fondos externos, una fuente tradicional para el desarrollo agropecuario y rural durante los 1960s, 1970s y 1980s provino de los organismos financieros internacionales como el BID y el Banco Mundial, y de organismos de ayuda internacional. Esas fuentes han perdido peso a nivel global 
y para la región, tal como se mostró en la presentación marco de la sesión (ver Gráfico 1 que muestra como la asistencia para el desarrollo neta ha caído como porcentaje del PBI mundial, de los países en desarrollo, y de ALC).

\section{Grafico 1: Asistencia para el Desarrollo y Ayuda Oficial Neta (\% del PIB)}

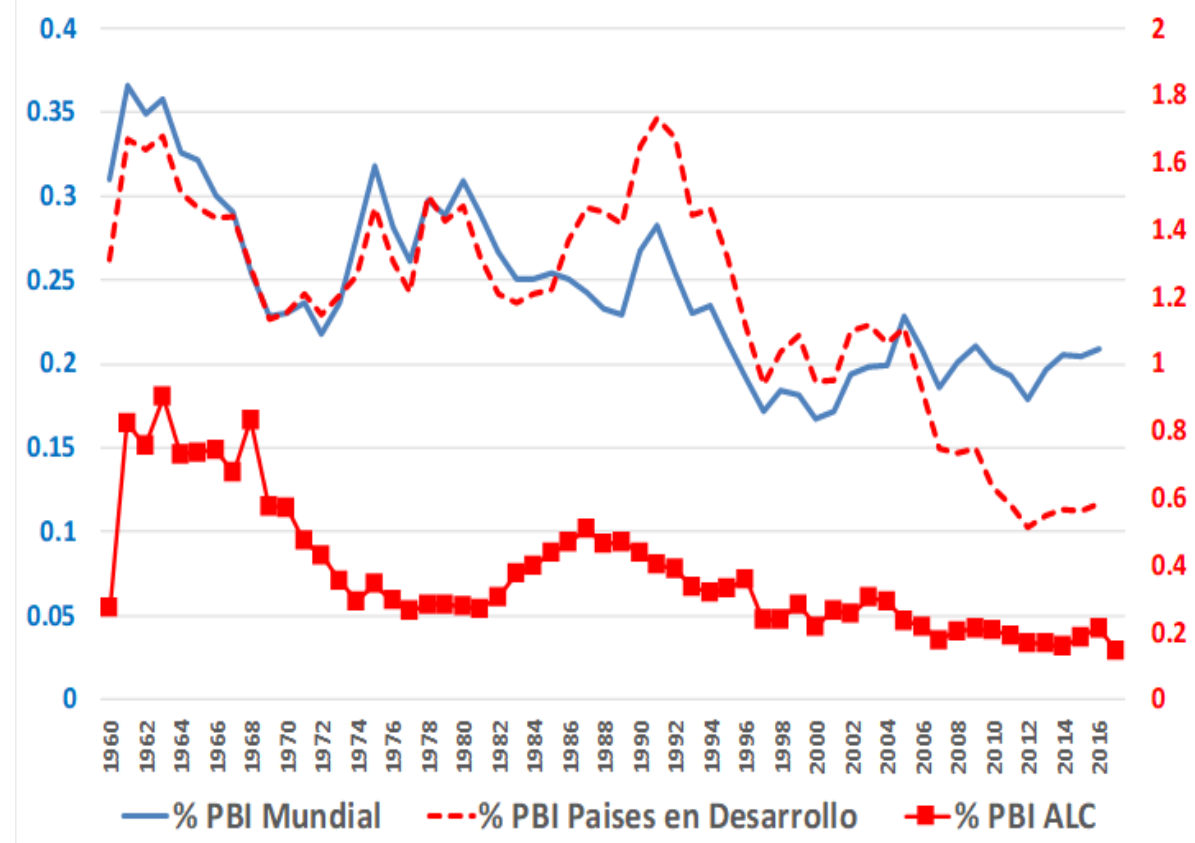

Fuente: World Development Indicators, World Bank

Por su parte, los préstamos no concesionales de bancos multilaterales (en flujos netos) son aún menores: en 2015, el total de préstamos netos fue casi 23,000 millones de dólares $(0.08 \%$ del PIB de los países en desarrollo; el BID en el período 2013-2017 prestó (en flujo neto) un promedio de 3,400 millones de dólares por años (0.06\% del PBI de ALC en promedio en esos años).

Además, el porcentaje de la ayuda para el desarrollo y de los préstamos no concesionales que era orientado al desarrollo agropecuario y rural también se fue reduciendo, aunque, como notaron en la discusión, esa reducción puede ser menor si se considera que una parte del financiamiento hacia el sector agropecuario y rural puede estar fluyendo bajo otros rótulos (por ejemplo, como parte de "blended finance").

\subsection{Remesas y fondos externos privados}

Un caso que se mencionó es el de una cooperativa de Guatemala que financia algunas actividades de sus socios en parte con remesas que provienen del exterior. 
Otra fuente de fondos mayormente externa es la que corresponde a inversores de impacto u otras organizaciones filantrópicas, que pueden aceptar rendimientos menores a cambio de la obtención de ciertos objetivos sociales o medioambientales. En la presentación marco se mostró que los fondos disponibles son significativos (ver Cuadro 1) y pueden convertirse en una fuente importante de financiamiento.

\section{Cuadro 1: Indicadores de Riqueza Privada y de Mercado de Capital}

\begin{tabular}{|l|l|}
\hline Riqueza Total de las Familias (2015) & $\begin{array}{c}250 \text { miliones de } \\
\text { millones }\end{array}$ \\
\hline Inversores de Impacto & \\
\hline Bajo administración (2017) & 228,000 millones \\
\hline Flujo de operaciones (2017) & 35,500 milones \\
\hline Bonos verdes (emisión en 2018) & 167,600 millones \\
\hline
\end{tabular}

Fuente: Díaz-Bonilla y Saravia-Matus, 2019

En este caso se mencionó varias veces que una limitación para poder acceder a estos recursos es la falta de proyectos con la relación adecuada de beneficio y riesgo. Esto se lo relacionó, no con la falta de oportunidades objetivas de inversión, sino con la falta de mecanismos de articulación y de preparación de operaciones que puedan desarrollar y presentar esos proyectos (o grupos de proyectos) a potenciales inversores privados. En la presentación marco se argumentó que dadas las bajas tasas de interés a nivel mundial (ver Gráfico 2), proyectos de desarrollo agropecuario, rural o del sistema agroalimentario adecuadamente estructurados pueden ofrecer alternativas atractivas para los fondos privados mencionados en el Cuadro 1.

Grafico 2: Tasas de Interés (nominal, anual) 


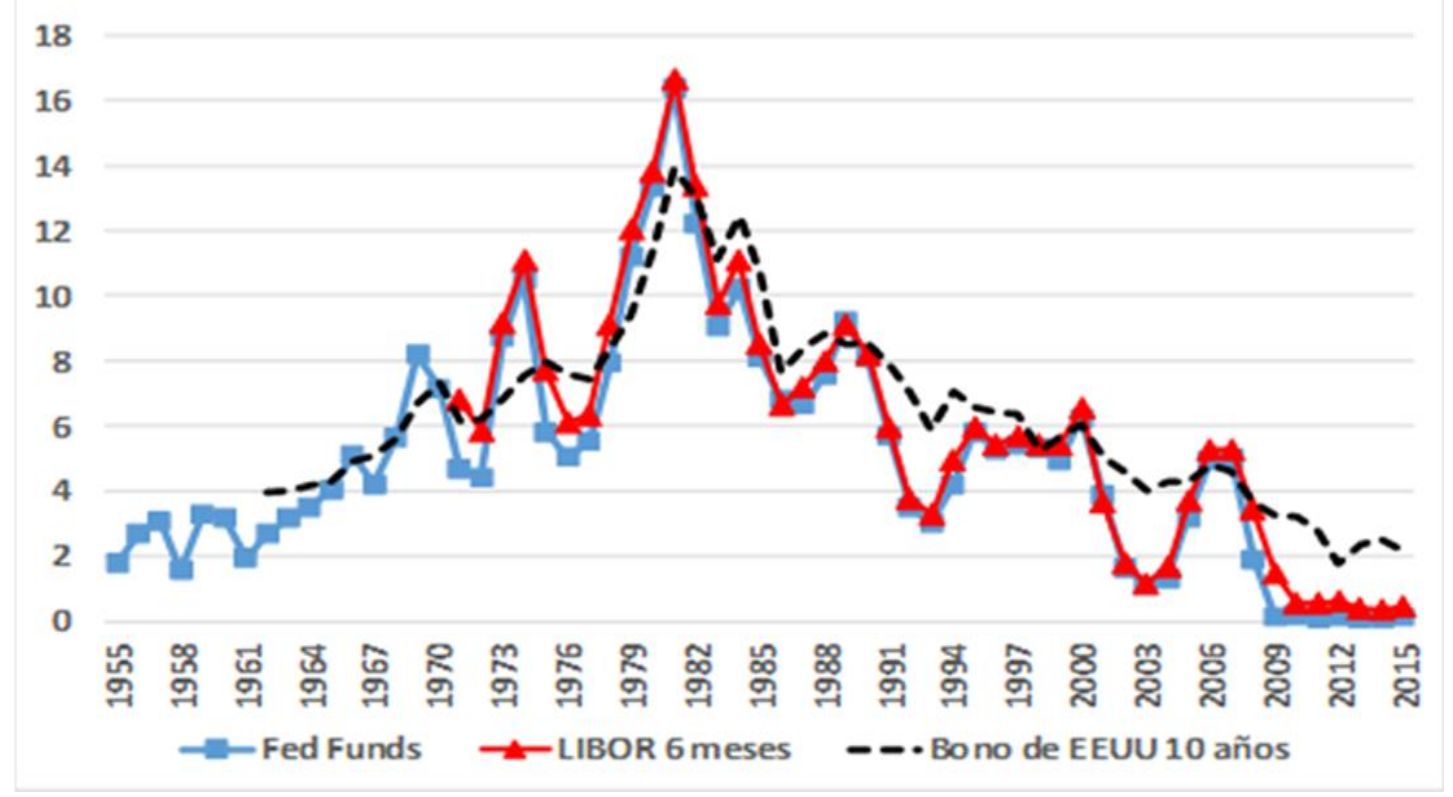

Fuente: U.S. Federal Reserve

\subsection{Otros aspectos relacionados con fuentes de fondos}

Un aspecto relacionado fue la discusión sobre la falta de mecanismos como incubadoras y aceleradoras de proyectos adaptadas al sector agropecuario (donde se puede requerir una perspectiva de más largo plazo que las inversiones de capital de riesgo tradicionales).

Se mencionó brevemente el tema de "fintech," "crowd-funding" y aspectos similares en los que se pueden usar medios digitales para generar una intermediación directa entre ahorros privados y potenciales utilizadores o inversores de esos recursos. Un aspecto limitante que se discutió es la falta de confianza por parte de depositantes, inversores, y otros posibles participantes, en parte relacionado con la escasa regulación y supervisión de esas actividades.

Más en general, las regulaciones afectan también el atractivo relativo de las diferentes formas de fondeo. Ya se discutieron las regulaciones macro-prudenciales en la sección 3. Pero hay otras regulaciones de tipo más microeconómico e institucional. Una regulación inadecuada puede impedir el desarrollo y la innovación financiera.

\section{INSTITUCIONES FINANCIERAS Y REGULACIONES}


Hay una gran variedad de bancos y de instituciones financieras, así como de operadores formales e informales que dan crédito, manejan ahorros, y ofrecen otros servicios financieros a la población rural y al sistema agro-alimentario en general. Por ejemplo, hay bancos públicos de desarrollo agropecuario (de primer y de segundo piso); bancos comerciales; cooperativas de ahorro y crédito; bancos comunitarios y comunales; instituciones formales de microcrédito; fondos de capital de riesgo de diferentes tipos; ONGs e instituciones benéficas; agroindustrias, proveedores de insumos y supermercados; y una variedad de prestamistas informales.

Cada tipo de institución financiera tiene su propia lógica para dar crédito, manejar ahorros y ofrecer servicios financieros a la población rural; cada uno tiene ventajas y limitaciones a analizar.

Por ello se plantearon las siguientes preguntas:

¿Cómo es la estructura del sistema financiero rural en su país, qué necesidades y clientes atiende, cuán eficiente y sostenible es? ¿Qué rol puede haber para la banca pública?

¿Hay ejemplos de arreglos institucionales que sean particularmente exitosos (o lo opuesto)? ¿Hay regulaciones micro- institucionales que sean particularmente negativas (o positivas)?

\subsection{Bancos públicos y privados. Primero y segundo piso.}

Los bancos públicos de desarrollo con orientación agropecuaria pueden ser un instrumento poderoso, pero han sido criticados muchas veces por fallar en movilizar el ahorro rural y ofrecer otros servicios financieros rurales; por ser ineficientes; y por corrupción o ser capturados por intereses privados. En el foro se mencionó la necesidad de analizar la economía política de la banca pública. Esas instituciones son en muchos casos consideradas por los productores como logros obtenidos por sus esfuerzos de organización y petición (“lobby”) a las autoridades, y los cambios en las mismas están dominadas muchas veces por el ciclo político sin la orientación de largo plazo que deberían tener. Se mencionó que en Perú, por ejemplo, el banco público agrario está quebrado pero que lo mantiene porque si no se va a crear otra entidad equivalente.

Por otro lado, luego de que muchos bancos públicos fueran cerrados en la región, los bancos privados no necesariamente llenaron el vacío. Algunas experiencias internacionales de bancos de desarrollo reformados y nuevas ideas sobre cómo rediseñarlos para no caer en los problemas del pasado sugieren la necesidad de profundizar el análisis sobre este tipo de institución.

Hubo también una discusión sobre si la banca pública debe ser de primer o de segundo piso, o si se necesitan los dos tipos dentro de cada país. Los bancos tradicionales de primer piso son vulnerables a 
captura y presión política para asignar crédito y hacer cumplir compromisos de pago. Los bancos de segundo piso son vulnerables a ser capturados por bancos comerciales y no tener control de la asignación de crédito.

Varios participantes argumentaron que es difícil usar a los bancos comerciales para llegar al productor (lo cual limita a la banca pública si fuera solamente de segundo piso). Un participante argumentó que en su país la banca privada se abstiene de aportar recursos porque implican riesgos que están penalizadas en las regulaciones. El sector privado entra y sale dependiendo de las oportunidades y de si las épocas son buenas o malas. Los bancos privados comerciales tienen potencialmente gran capacidad, pero con un sesgo hacia el mayor retorno comercial de zonas urbanas y grandes agricultores. La regulación puede aliviar ese sesgo pero a la vez generar distorsiones que pueden ser costosas.

En el caso de Colombia se mencionó que para lograr que bancos de primer piso lleguen a la producción primaria y a pequeños productores se están dando garantías que pueden llegar hasta el $80 \%$ del préstamo (lo que se consideró excesivo). En el caso del FIRA se mencionó que como una institución de segundo piso trabaja no solamente con bancos comerciales sino con otras instituciones no bancarias.

Por su parte se discutió que la banca pública (de primer o segundo piso) tiene el problema de cómo combinar los aspectos de fomento al desarrollo y de sustentabilidad comercial. El tema se centra en cómo compatibilizar el manejo de riesgos con el objetivo de desarrollo. Un participante mencionó que usar la mantención de capital como una métrica de resultados genera en los funcionarios públicos aversión al riesgo ya que están bajo la amenaza de recibir evaluaciones negativas de las organizaciones públicas de auditoría y control. En el caso de su organización se regresó a usar la métrica de personas atendidas.

\subsection{Cooperativas, bancos comunitarios y microcrédito}

Las cooperativas de ahorro y crédito y los bancos comunitarios y comunales tienen conocimiento y orientación rural pero son débiles en escala y gestión. Las instituciones de micro-financiamiento pueden aportar en muchos contextos, pero no tienen escala para financiar la reconversión de sistemas agroalimentarios, y en algunos casos, sus estructuras financieras no son necesariamente sostenibles

Además, las regulaciones pueden ir en su contra. Se mencionó que en Ecuador la ley de la Economía Popular y Solidaria permitía el desarrollo de instituciones financieras más comunitarias, como el llamado Fondo Populorum Progressio, pero que ese fondo fue obligado a bancarizarse debido a la ley de Entidades Financieras. En ese país también existen cooperativas que tienen más amplia cobertura y 
volumen de financiamiento que el banco público de primer piso. Mientras que el mismo marco de políticas para el sector solidario y el sector informal facilitaba la operación de esas cooperativas, la ley financiera obligó a muchas de esas cooperativas a formalizarse. Por su parte, el representante de la cooperativa de Guatemala con 4500 productores mencionó que también operan como entidad financiera pero que no son parte del sistema financiero regulado.

\subsection{Otras instituciones e intermediarios financieros}

En el caso de Chile se mencionó que hay fondos públicos de capital de riesgo, los que a su vez apalancan fondos privados.

Otras instituciones incluyen fondos internacionales de inversión con objetivos sociales (como Oikos que estaba representado en la reunión) y que trabajan y asumen riesgos con productores pequeños.

Se discutieron también otras instituciones o agentes, tales como la agroindustria que hace de ancla en las cadenas de valor o empresas proveedoras de insumos como potenciales canales de financiamiento. Pero se mencionó la falta de regulaciones para que esas entidades sean candidatos naturales para proveer crédito, así como la falta de acceso a oportunidades de "blended finance." Por otra parte, esas entidades en varios casos trabajan principalmente con empresas grandes, y hay diferentes visiones respecto de si pueden o quieren trabajar con pequeños productores.

Otra institución son los diferentes fondos públicos de garantías que son parte de los sistemas financieros de muchos países de la región. Se los mencionó en el contexto de los problemas económicos de los sectores lechero y arrocero en Uruguay, y cómo se crearon fondos de garantía para flexibilizar la reprogramación de sus deudas.

También se discutió el papel de otros agentes o entidades no afiliados a los bancos pero que pueden proveer otros servicios financieros, como corresponsalías para medios de pago y otras transacciones. Se dijo que la posibilidad de esos desarrollos "llegan solamente hasta donde la regulación los empuja." Previamente se mencionó el posible papel de incubadoras, aceleradoras, y diferentes esquemas de "fintech" y "crowd-sourcing."

Se debatió si la regulación traba la innovación. Se analizó la necesidad de tener mecanismos de experimentación regulatoria (un "sandbox" regulatorio), que sirvan como laboratorio para la innovación regulatoria, analizando cómo podrían funcionar operaciones del sector privado en el sector público para manejar el riesgo y salir de las reglas usuales. 


\section{MANEJO DEL RIESGO EN LAS ENTIDADES FINANCIERAS}

Hubo una detallada presentación por parte de uno de los fondos privados de microcréditos que participó del foro sobre cómo identificar el riesgo y cómo manejarlo en instituciones financieras, considerando al menos tres niveles: individual de clientes; la cartera de la institución; y el riesgo más sistémico. Lo que sigue está basado directamente en esa presentación.

\subsection{Riesgos individuales}

A nivel individual, se indicó que era un error el enfoque común de mucha banca estatal de basarse en proyectos por productos (maíz, lechería, etc.) como si fueran independientes del entorno del productor. Se argumentó que una lección ha sido la necesidad de analizar el riesgo viendo la totalidad del flujo económico del hogar, y no solamente el proyecto a ser financiado, ya que la unidad familiar es la principal administradora del riesgo propio. Por ejemplo, un tema es si los clientes (su unidad familiar) están suficientemente diversificado para que la cartera sea menos riesgosa. Las microfinanzas han aprendido a mirar y financiar la globalidad del cliente, la totalidad de su empresa, viendo cómo se insertan en las cadenas de valor y otras actividades.

De todas maneras, este análisis es solamente el principio, ya que las unidades familiares y sus riesgos son muy heterogéneos; como cada cliente es distinto, hay que conocerlo a cada uno individualmente. Hay fallas de mercado y el punto inicial es la falta de información. Desde una visión urbana todos los productores son iguales. El problema es tratar igual a los que no lo son. Ese análisis individual sirve para definir el nivel de crédito y servicios financieros y además, en el caso de instituciones de desarrollo (se mencionó el INDAP de Chile), puede servir para definir quiénes pueden ser graduados del sistema público. Como se nota más adelante, la revolución digital puede ayudar analizar los perfiles individuales y de familias para entender mejor la heterogeneidad demográfica y geográfica, y diseñar e implementar productos y servicios financieros que consideren dicha heterogeneidad a un bajo costo.

La pregunta desde la perspectiva de la entidad financiera es "cómo hago para aprender acerca de cada uno de mis clientes a un costo razonable." Se pueden bajar los costos de la evaluación de riesgo usando tecnología de la información y las comunicaciones. Pero se requiere encontrar un equilibrio entre herramientas digitales y la relación personal. Una digitalización total elimina el necesario contacto personal. Por eso una práctica apropiada es tener una visita al cliente al iniciar la transacción, usando una tableta para recopilar toda la información del cliente, incluyendo aspectos productivos como suelos. Se necesita un diálogo para entender el flujo de fondos de la familia. Sobre esa base se hace un 
contrato individualizado definiendo cómo se hacen los desembolsos y pagos. También se ofrece una cuenta de Facebook para que los dientes interactúen.

En este sentido es importante asegurar que ese mejor conocimiento tenga valor para la entidad y para el cliente. Esto requiere tener relaciones de largo plazo y compartir con el cliente los beneficios que surjan de esa relación a través de mejores plazos, tasas y otras condiciones. Por otra parte, crear relaciones y sostenerlas requiere tener un buen manejo del oficial de crédito, porque si éste se va, se puede ir con el cliente. Un punto que fue mencionado es que si bien la tecnología digital permite un mayor acceso a la información del cliente, no se debe permitir que reemplace el contacto personal con el cliente, que tiene valor en sí mismo para crear confianza y reducir riesgos.

La distancia con el cliente es un problema para la banca pública agropecuaria de segundo piso. La banca estatal no necesariamente resuelve las fallas de mercado más importantes (como los de la información).

\subsection{Riesgo de cartera y riesgo sistémico}

El segundo nivel es el manejo del riesgo de cartera, para lo cual hay que usar diversificación y ejercicios de simulaciones con diferentes tipos de productores (por ejemplo si son consumidores o productores netos; si tienen o no depósitos, etc).

El tercer nivel es el riesgo sistémico, que es un tema del gobierno. Se requiere seguridad jurídica para hacer valer el contrato. Hay carencia de infraestructura, de tecnologías, de asistencia técnica, de seguros adecuados y de asociaciones sólidas de productores y esto no se resuelve con la banca pública. Hay que entender cuáles son las barreras principales para el acceso al crédito y los servicios financieros, que pueden o no ser resueltos con la banca desarrollo. Instrumentos como los bonos por desastres pueden ayudar.

En general, se enfatizó la importancia de la gestión integral de riesgos, que complementen instrumentos para atender diferentes problemas (tales como riesgo de mercado, riesgos productivos y riesgos sistémicos).

\section{PRODUCTOS Y SERVICIOS FINANCIEROS}

Varios de los sesgos anti-rurales del sistema de crédito tradicional, y su foco en comercialización y procesamiento, pero no en inversión, se originan en la dispersión y baja escala de los clientes, así como riesgos covariantes (clima, precios, plagas, estacionalidad de la producción). Pero también hay otras 
razones, incluyendo regulaciones diseñadas para el sector urbano y pensando en actividades con flujos de caja más regulares que el sector agropecuario. Asimismo, faltan otros productos y servicios financieros para la población rural, más allá del crédito.

Por ello, para esta sección, se plantearon las siguientes preguntas:

¿Qué tipo de productos financieros existen en su país, por el lado de los activos y pasivos del sistema bancario/financiero?

¿Cuál es el valor/volumen de aplicación de esos instrumentos?

¿Hay algunos que sean particularmente útiles (inútiles) para los objetivos buscados? ¿Qué tan extendido está el uso de los mismos? ¿Qué habría que hacer para expandir el uso de los instrumentos útiles (limitar el de los inútiles) sin provocar problemas mayores?

¿Qué tipos de enfoques y modos de entrega/interacción con los clientes existen? ¿Alguna idea de la dimensión de su uso? ¿Qué funciona y qué no funciona para lograr mayor inclusión financiera, especialmente de grupos marginados y vulnerables?

\subsection{Crédito de largo plazo}

Una carencia obvia mencionada en el debate es la de crédito de inversión de largo plazo. En la banca privada, o la pública también si el fondeo es con depósitos de clientes, este tipo de instrumento tiene limitaciones debido a descalces importantes con la estructura de pasivos del banco (es decir los depósitos de los clientes). El desarrollo de crédito para inversión de largo plazo requeriría fondeo de fuentes fiscales o monetarias públicas, o la intermediación en el mercado de capitales. Pero esos programas públicos de fondeo de largo plazo muchas veces se mueven de acuerdo al gobierno de turno, cambiando productos y condiciones.

Hubo discusión sobre otros productos financieros, tales como arrendamiento con opción a compra (“leasing"), recibos de almacenamiento ("warrants"), prendas de productos, préstamos a cadenas de valor, préstamos proveedores de insumos, la cesión de contratos de venta, el descuento de facturas y otros.

\subsection{Leasing, warrants y prendas de productos}

Respecto del "leasing" ya se mencionó la limitación de las pocas empresas que existen para fondearse en el mercado de capitales, y por ende deben utilizar el crédito bancario, lo que sube el costo y limitan 
la competitividad de ese instrumento de financiamiento. Respecto de warrants se dijo que faltan certificadores o son caros y por tanto no se han desarrollado tanto. Se mencionó el caso de Ecuador, donde en los 1990s se crearon certificados de depósitos para granos básicos, pero hubo problemas con la certificación de los almaceneros, fallaron los controles, y al menos una parte del producto físico fue vendido separadamente por los productores.

Problemas similares pueden darse con la prenda de productos, cuando los productores terminan vendiendo a otros posibles compradores con mejores precios. Pero en Uruguay se mencionó el caso de fideicomisos ganaderos donde el banco tiene propiedad del ganado con trazabilidad.

\subsection{Préstamos a cadenas de valor}

En lo que hace a préstamos con garantías contra contratos en cadenas de valor se argumentó que, aunque bajan objetivamente el riesgo, esto puede no ser reconocido por las entidades financiera debido a regulaciones (que igual obligan a tomar previsiones muy altas), o puede haber dificultades para diseñar mecanismos de cobro por parte de los agentes de la cadena de valor que compran los productos, y que puedan hacer retención de pagos para cubrir los requerimientos de los bancos que financian.

Se mencionó que en varios casos el financiamiento por cadenas se focaliza más en aquéllas formadas por productos de exportación que en las que corresponden a alimentos domésticos, que son claves en los sistemas agro-alimentarios.

En todo caso se consideró que esta opción tenía un gran potencial para desarrollarse en la región.

\subsection{Garantías, pagos flexibles y préstamos con insumos}

Hay otros temas de regulaciones que pueden afectar el uso de ciertos instrumentos, tales como cómo se valorizan las garantías, y cómo se reconoce o no la pérdida para que el intermediario pueda recibir la cobertura por garantía.

Hubo también una discusión acerca de maneras de ajustar tanto los desembolsos como los pagos al ritmo productivo más irregular y variado de las actividades agropecuarias. Pero las regulaciones (por ejemplo, sobre la regularidad de los pagos) pueden limitar esta opción.

Se mencionó el caso de Uruguay y los préstamos ganaderos, en los cuales los desembolsos son en función del proceso productivo. 
También se habló de la posibilidad que los préstamos que se puedan realizar en insumos. Desde el punto de vista de los pagos, se comentó la posibilidad de mecanismos de pago alineados con el ciclo agropecuario, y, más específicamente, créditos que puedan ser pagados con productos. Se mencionó un esquema de seguros en la Argentina en el cual las pólizas se pueden cobrar en granos en kilos de soja, que se acopian y luego son vendidos por la empresa de seguros usando los mecanismos normales de comercialización. Se indicó que los productores están más acostumbrados a pagar en producto (como hacen con el arriendo de tierras). La empresa aseguradora puede tomar posiciones en el mercado de futuros para fijar el precio y sus ingresos. Como contraejemplo, se mencionó el caso de Ecuador donde hubo una experiencia de pagar en especie, pero que fue un fracaso ya que no había experiencia de parte de los bancos y sus agentes no sabían la calidad de lo que les estaban entregando como pago. También se mencionaron casos en El Salvador con en el Programa Mundial de Alimentos, donde se entregaban granos de peor calidad; o el tema de los arroceros en Colombia, que pagan en especie, pero el tema es quién define el precio del producto.

Idealmente, podría pensarse en un mecanismo de pago contingente relacionado con el precio y cantidad de los productos financiados; viceversa, el camino más común es mantener esquemas fijos de pago, y pensar en diferentes formas de seguros para el productor (aunque también podría pensarse en seguros para el banco).

\subsection{Seguros}

En este sentido un tema central es el de seguros y manejo del riesgo, como otro aspecto de los servicios financieros que necesita la población rural y los agentes de los sistemas agro-alimentarios.

Se mencionaron diferentes variantes tales como microseguros y seguros basados en índices climáticos. Se comentó también el tema de seguros que existen pero que no se toman, y la pregunta es por qué. Un tema relacionado fue el de regulaciones que pueden trabar la innovación en esquema de seguros, como el caso de seguros paramétricos para eventos climáticos, pero que en el caso de un país la Superintendencia consideraba el pago como ganancia indebida.

Otro instrumento mencionado que se lo consideró importante sería el seguro de ingresos, pero no hay ejemplos en la región.

Se señaló que falta un análisis sobre el impacto que pueden tener los seguros: por ejemplo, ¿bajan las tasas en créditos asegurados versus los no asegurados?; ¿solamente afecta el acceso? Se plantearon estas preguntas como temas por estudiar. 


\subsection{Otros instrumentos y servicios financieros}

Se reconoció que además de créditos (y seguros) existe una variedad de servicios financieros que son importantes para la población rural y los productores agropecuarios, tales como depósitos (cheques y cuentas de ahorro), mecanismos que faciliten los pagos, y otros servicios. En general, estos servicios aumentan los fondos prestables y mitigan riesgos. Pero se mencionó que regulaciones que vinculen el ahorro y crédito rurales pueden también tener resultados indeseables: si un mínimo de ahorro debe canalizarse a crédito rural, o un mínimo de crédito debe provenir de ahorro rural, tanto el ahorro como el crédito pueden deprimirse.

\subsection{Uso de tecnologías digitales}

Se resaltó la promesa de la tecnología digital (internet, telefonía celular, agencias rodantes, y otros) como opción para bajar costos de transacción y para generar más información sobre posibles clientes (bajando el riesgo). Pero se preguntó por qué esa promesa no parece haberse capitalizado en mayores servicios o en rebaja de costos.

Se reconoció que para eso hace falta infraestructura, que los posibles clientes tengan acceso a computadoras, teléfonos, y que haya educación financiera. Sin una "carretera" de internet no hay infraestructura para medios de pago electrónicos y no se van a desarrollar las "Fintech" en el sector rural.

\subsection{Inclusión financiera y otros instrumentos}

Un aspecto relacionado discutido fue el de la inclusión financiera. Se dijo que a veces se queda en las metas del gobierno como el número de cuentas de ahorro, pero no se analiza su uso. En muchos casos, no tienen mayor utilización. Las familias que reciben transferencias condicionadas usan las cuentas de ahorro solo para recibir el dinero que entra y que inmediatamente se retira. Por ejemplo el banco agrario en Colombia es el pagador de transferencias sociales, lo que ha llevado una mayor inclusión financiera, medida como cuentas bancarias, pero luego no son aprovechadas. No se usan como ahorro. Se mencionó la importancia de la educación financiera (por ejemplo, sobre cómo leer estados financieros y "vouchers," como presupuestar, cómo usar los cajeros automáticos) y se dio el ejemplo de un proyecto con mujeres que luego de recibir educación financiera dejaron el $16 \%$ del dinero como ahorro, cuando antes de dejaban solamente el $2 \%$.

Hubo un debate sobre que el tema de las finanzas agropecuarias es diferente del de inclusión financiera, y que este tema está generalmente ausente en la discusión. 
Un tema relacionado discutido fue si no era preferible usar otros instrumentos diferentes del crédito para atender a los productores pobres y vulnerables. Quizás en estos casos es mejor usar transferencias de las redes de protección social ampliadas con fondos de apoyo para aspectos productivos, y otros servicios de apoyo complementarios como salud.

Se discutieron las limitaciones para mujeres en los mercados financieros que afrontan barreras mayores para acceder a crédito y otros servicios, debido a aspectos relacionados con la propiedad tierra, capacidad legal para entrar en contratos, requerimientos de permisos de los maridos, y problemas similares.

Se comparó el tema de pagos electrónicos en África que fue desarrollada por las empresas telefónicas (como MPesa en Kenia), pero que no daban información de costos administrativos. En la región hay ejemplo de medios de pagos armados por empresas telefónicas en el Salvador, Paraguay, y Haití. Pero sigue habiendo desconfianza en los medios de pago electrónicos, y limitaciones por falta de conexión telefónica. Sin embargo, estos mecanismos están desarrollándose entre las poblaciones más jóvenes que pagan con celulares.

Finalmente hubo un breve análisis acerca de si se necesitan instrumentos especiales para apoyar una mejor nutrición, cadena de valor más cortas, productos que pueden ayudar al desarrollo a los mercados locales. Se mencionó el caso de apoyo a la comercialización de pequeños productores con un sistema de becas que financian producción de alimentos saludables y que tienen que ser comprados a productores apoyados por el INDAP. Otros aspectos son el financiamiento de compras a productores para abastecer sistemas escolares y a la agroindustria para producir productos mejores desde el punto nutricional.

\section{PRODUCTOS Y SERVICIOS DE APOYO AL FINANCIAMIENTO}

En la reunión también se discutió la importancia de articular el crédito y otros instrumentos y servicios financieros, por una parte, con productos y servicios no financieros, por la otra, para mejorar el acceso al crédito y la inclusión financiera, a la vez que pueden ayudar a una mejor asignación de los recursos y a reducción del riesgo.

Aspectos financieros ya mencionado son el de seguros (tradicionales, microseguros, seguros basados en índices climáticos, sistemas que usan fotos de celulares, etc), y los fondos de garantía de crédito. 
Entre los productos y servicios no financieros existe una variedad a considerar. Ya se discutió la importancia de la educación y capacitación financiera (la que necesariamente tiene que considerar, entre otras cosas, los productos y servicios financieros ofrecidos). Se sugirió que la misma debía incluir (o quizás empezar) por los representantes políticos y gremiales (que son los que muchas veces presionan por perdones de deudas cuando lo que se necesita es simplemente una restructuración del pago), y no solamente para los productores más pobres.

Otro tema de gran importancia es el catastro de tierras, que se consideró particularmente importante en el caso del manejo del tema de tierras en el post conflicto en Colombia. También se enfatizó la importancia de la inversión en riego, $1+\mathrm{D}$ agropecuaria (junto con extensión), mejor información de mercado y clima, sanidad animal y vegetal, infraestructura en general (caminos, electricidad, telecomunicaciones...), sistemas de meteorología, sistemas judiciales eficaces, y seguridad ciudadana en zonas rurales. También ayudaría reforzar la asociatividad de los productores y fortalecer esas asociaciones. Un aspecto relacionado es el desarrollo del mercado de servicios privados de diferentes tipos en el medio rural, utilizando tecnologías de comunicaciones, los que pueden también atraer a la juventud. Como se dijo esto requiere desarrollar la infraestructura de telecomunicaciones e internet para poder usar diferentes servicios digitales, financieros y no financieros.

Relacionado con la seguridad ciudadana en zonas rurales, también se mencionó la importancia de controlar el lavado de dinero (muchas veces relacionado con comercio de drogas) con sistemas de seguimiento en medios rurales.

Un servicio importante puede ser el de asistencia técnica tanto para que los productores y asociaciones puedan identificar proyectos y como para los bancos para que puedan aprovechar esas oportunidades. En general un tema de investigación es cómo desarrollar la asistencia técnica y los servicios para abrir mercados y desarrollar negocios, y las diferentes formas de organizarse y financiar esas actividades.

\section{POLÍTICAS PÚBLICAS}

Se dedicó una sesión al tema de políticas públicas en general. Se plantearon varias preguntas:

¿Cómo es el marco de políticas públicas en su país relativo al desarrollo financiero rural y cuáles son sus carencias y falencias principales? ¿Qué rol tiene la banca pública y cómo podría mejorarse?

¿Qué aspectos de las discusiones de política pública en sesiones anteriores le parecieron de mayor importancia y aplicabilidad para recomendar reformas en el su país? 


\section{¿Cómo se podría mejorar el conocimiento y profundizar el análisis en su país sobre los temas claves para producir una propuesta de política pública para el mercado financiero rural?}

En la presentación marco (ver también Díaz-Bonilla y Fernández-Arias, 2019) se mencionó que las fallas de las políticas públicas de desarrollo del pasado basadas en la represión financiera y los créditos públicos ilustran cómo las distorsiones introducidas pueden hacerlas globalmente contraproducentes. ¿Cómo evaluar la justificación y bondad de una política? Hay tres niveles básicos de análisis:

1) Falla de mercado. Identificar con precisión dónde el mercado falla en incorporar la rentabilidad social (es ineficiente) o el objetivo social perseguido que motiva la política.

2) Diseño de políticas. Demostrar que la política está diseñada para tratar específicamente la falla identificada (controlando los efectos secundarios)

3) Implementación de políticas. Verificar que la implementación se ajusta a las capacidades institucionales (ser consciente de las fallas de gobierno)

Respecto de los tipos de políticas públicas, en la presentación marco (ver también Díaz-Bonilla y Fernández-Arias, 2019) se mencionó que hay que distinguir dos clases: a) la provisión de insumos públicos (complementando a los agentes privados con infraestructura, agencias públicas, etc); y b) intervenciones de mercado (incentivando a los agentes privados con subsidios, impuestos, regulaciones).

También se suele distinguir el alcance de dichas políticas de acuerdo a si son horizontales o transversales (atienden a varios mercados, sectores, o productos), o verticales (dirigidas a sectores favorecidos).

El análisis de las políticas depende del tipo de políticas

La provisión de insumos públicos horizontales incluye una amplia gama de intervenciones que suelen tener clara justificación tales como una macroeconomía estable, infraestructura legal para el comercio y el crédito. La provisión de insumos públicos verticales, tales como la l+D agrícola y la asistencia técnica, son generalmente justificables si los beneficiarios solventes colaboran en la recuperación de costos.

Pero las intervenciones de mercado son más riesgosas y requieren más análisis para pasar los tres exámenes. Y las políticas verticales tienen más riesgos de captura que las políticas horizontales. Las intervenciones de mercado verticales (subsidio a cierto sector) son las más demandantes de capacidades institucionales para manejarlas.

Las intervenciones de mercado en el sistema financiero pueden estar orientadas a alterar el funcionamiento del sistema financiero (p.ej. garantías blandas para empresas productivas sin acceso al crédito) 
o utilizarlo como mecanismo (p.ej. subsidio a tasa de interés para préstamos a una actividad de interés social). El instrumento adecuado depende del fin.

En el debate se mencionó que además de no necesariamente seguir el proceso analítico mencionado más arriba, muchas veces las políticas públicas no son de largo aliento, sino que dependen del ministro o autoridades de turno y cambian muy frecuentemente. Créditos subsidiados cambian productos y esquemas con los gobiernos; los bancos públicos obedecen a los gobiernos de turno y no los objetivos de largo plazo como deberían.

Hay ejemplos de algunas instituciones como FIRA en México que se han mantenido como el único fideicomiso existente en el Banco Central y han estado relativamente al margen de vaivenes políticos. Por ejemplo, no se han dado subvenciones en contra de la sostenibilidad del fideicomiso.

En todo caso, como se mencionó anteriormente, es crucial la métrica de evaluación del banco público y quien evalúa. Una evaluación comercial del rendimiento financiero distorsiona el aspecto de desarrollo de la política y restringe el alcance del banco público. Hay una posible oposición entre autonomía para no ser dependiente del ciclo político e integración con otras agencias del gobierno para generar sinergias.

La agenda hoy es diseñar bancos de primer piso que atiendan fallas de mercado (complementarios del sistema privado) y ayuden a mejorar las políticas públicas, con fondeo trasparente e incentivos para buena gestión, y sujetos a regulación y supervisión bancarias

\section{SIGUIENTES PASOS: POSIBLES AGENDAS DE TRABAJO CONJUNTO}

Como cierre del evento se discutió la idea de generar un programa de trabajo futuro entre las personas e instituciones participantes que estuvieran interesadas. Ese futuro trabajo analizaría la experiencia a efectos de construir propuestas de reforma de políticas públicas e instituciones, pero también incluyendo desarrollos en actividades privadas:

*Actualizar el mapa de quién está proveyendo financiamiento agropecuario en la región (incluyendo financiamiento privado, financiamiento a cadenas de valor, y "fintech," entre otros temas). Tener un mapeo amplio.

*Mapear las políticas públicas de desarrollo financiero rural existentes y examinarlas en términos de justificación y efectividad, considerando las necesidades de inversión relacionadas con la transformación de los sistemas agro-alimentarios de la región. 
*Analizar la economía política de los bancos públicos de desarrollo, considerando autonomía versus integración, y los aspectos de gobernanza y diseño operativo de los bancos públicos de primer y segundo piso.

*Identificar los aspectos del marco regulatorio y macroprudencial del sistema financiero que pueden tener un impacto adverso particular sobre el desarrollo financiero rural "y las necesidades adicionales de inversión para la transformación de los sistemas agroalimentarios de la región, examinando si hay posibilidades para una reformulación.

Más allá de estos cuatro pilares de un estudio integral del desarrollo financiero rural, las discusiones apuntaron a una serie de temas de políticas públicas, tales como los siguientes, que es necesario analizar:

*Una agenda de investigación de políticas públicas y aspectos regulatorios para facilitar la innovación en la transferencia y manejo del riesgo

*Cuáles son las lecciones aprendidas en el uso de diferentes instrumentos, tales como el crédito subsidiado; quizá mejora el acceso, pero no necesariamente el nivel de desarrollo (productores que están recibiendo crédito por mucho tiempo y no se desarrollan).

*También se mencionaron como temas de análisis el estudio del efecto de los seguros sobre las tasas y los incentivos privados a prestar.

*Estudiar las lecciones aprendidas no solamente de crédito sino de otros instrumentos y servicios financieros y su articulación con otros servicios no financieros.

*Analizar la necesidad de revisar la regulación financiera para que sea sensible al valor de los seguros y se ajusta a las particularidades agropecuarias.

*El uso de tecnologías de la información y la inclusión financiera

*El desarrollo de plataformas como metodología de trabajo conjunto (el caso de Safin). Que esas plataformas funcionen como ecosistemas, quizá país por país, y no solo para intercambiar conocimientos sino también para apoyar inversiones concretas.

Es importante que las políticas propuestas estén basadas en evidencia, pero puede no ser suficiente por la economía política de cada caso. Hay que crear evidencia y compartirla. Evidencia de impactos. Hacer una agenda de seminarios para coordinar esfuerzos y difundir el trabajo. 


\section{ANEXO}

Durante el foro se presentaron los tres gráficos siguientes. El primero muestra el avance de las reformas financieras en varios grupos de países (el valor de 0 es no reformas y el valor de 1 es el de más reformas; ver la metodología en Abiad, Abdul, Enrica Detragiache, and Thierry Tressel. 2008 )

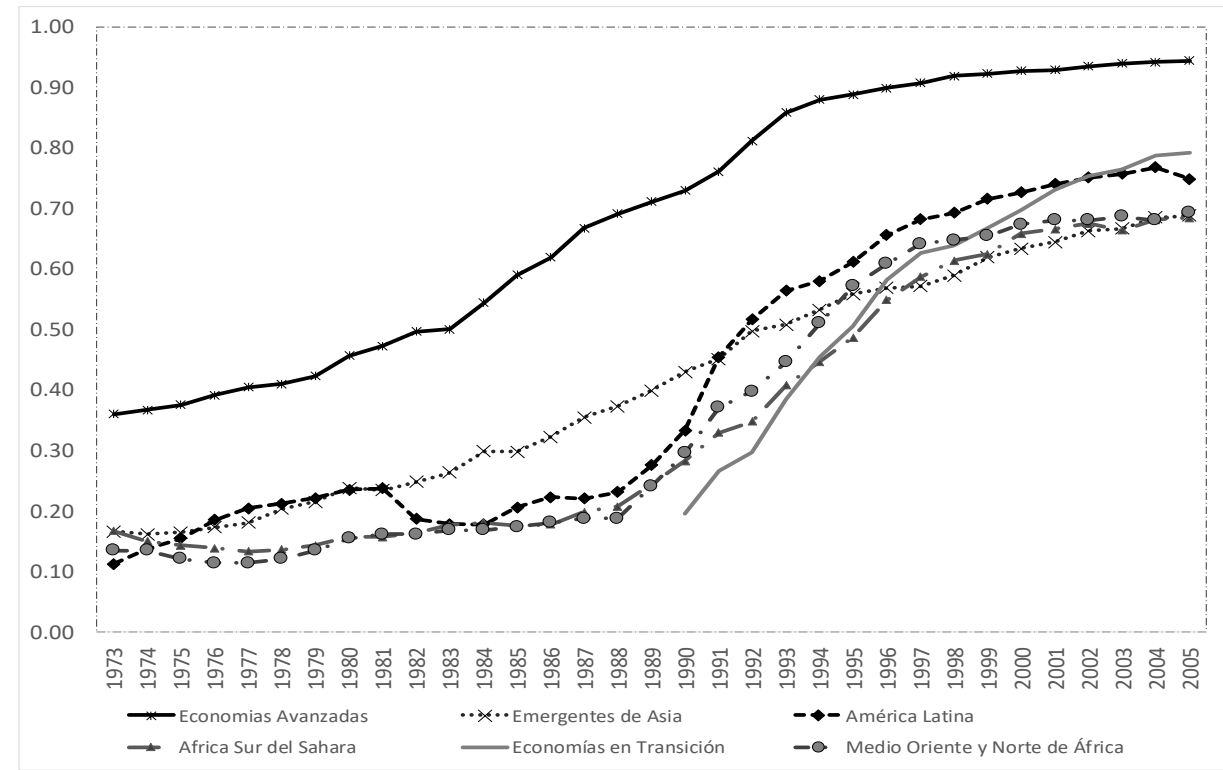

Fuente: Abiad, Abdul, Enrica Detragiache, and Thierry Tressel. 2008

El otro gráfico muestra el crédito al sector privado for regiones.

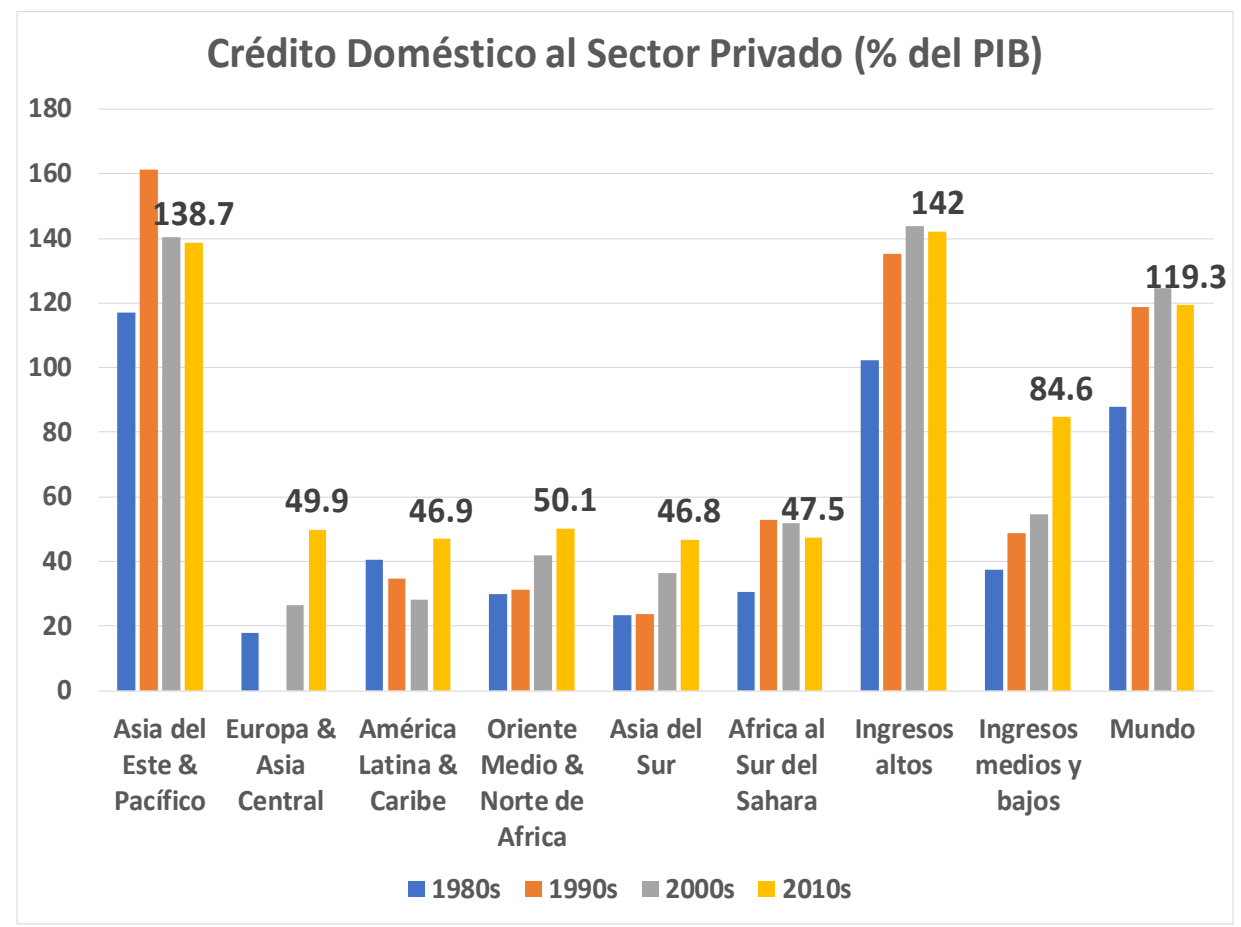

Fuente WDI/WB 
El tercer gráfico muestra el índice de orientación agropecuaria de ALC (década 2010s) comparado con otros grupos de países

\section{Indice the Orientación Agropecuaria \\ (Crédito)}

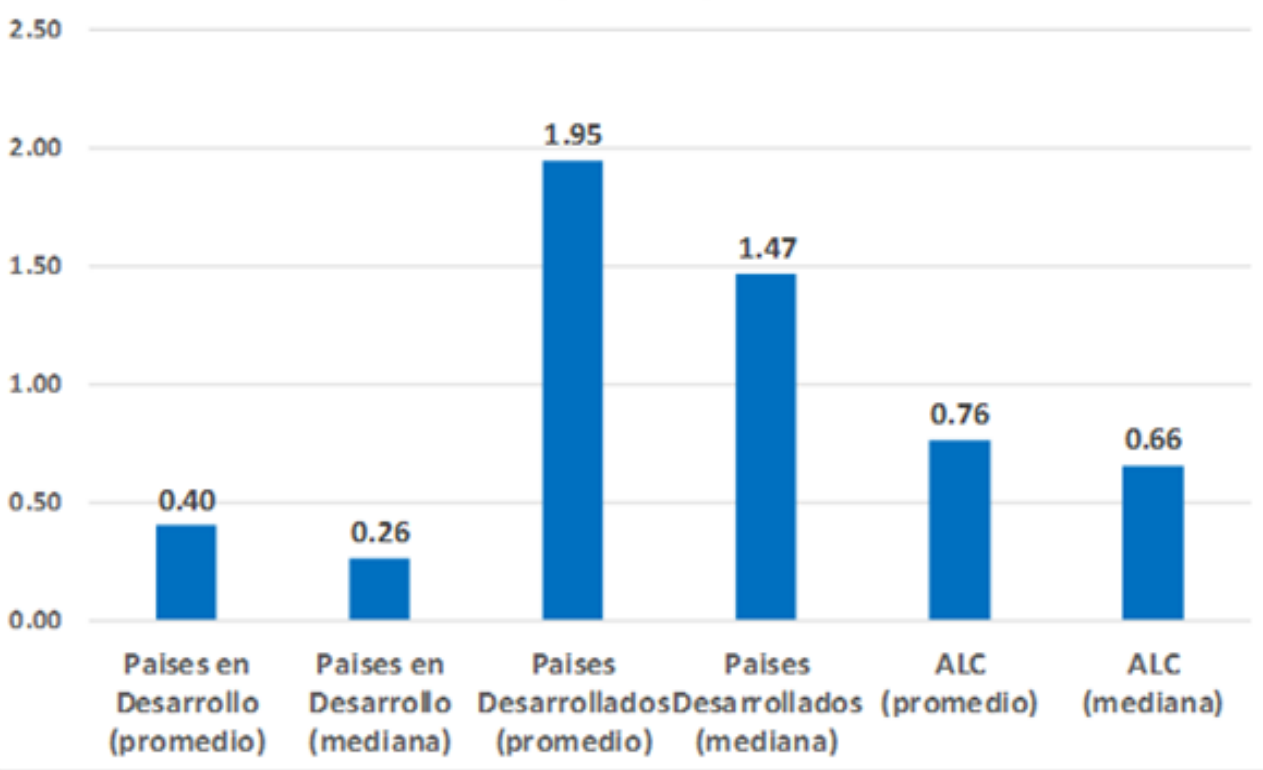

Fuente: FAOSTAT

\section{ABOUT THE AUTHORS}

Eugenio Diaz-Bonilla is the Head of Latin American and Caribbean Program at IFPRI.

Eduardo Fernandez-Arias is an independent international consultant.

Valeria Piñeiro is a Senior Research Coordinator with MTID at IFPRI.

Bettina Prato is Senior Global Engagement Specialist, IFAD

Joaquin Arias is Technical Specialist, IICA

\section{ACKNOWLEDGMENTS}

The authors express their appreciation for the substantive contributions from the participants in the conference "Financiamiento e inclusión financiera para el desarrollo agropecuario, rural y de sistemas alimentarios en ALC" that took place on May 6-7, 2019 at IICA Headquarters, San José, Costa Rica.

\section{REFERENCES}


Adams, D. W., D. H. Graham, and J. von Pischke, ed. 1984. Undermining Rural Development with Cheap Credit. Boulder, CO: Westview Press.

Díaz-Bonilla, E. 2017a. "Food Security Stocks: Economic and Operational Issues" Chapter 8 in Laborde and Bouet (eds) "Agriculture, Development, and the Global Trading System: 2000-2015." IFPRI Publications.

Díaz-Bonilla, E. 2017b. "Food Security Stocks and the WTO Legal Framework." Chapter 9 in Laborde and Bouet (eds) "Agriculture, Development, and the Global Trading System: 2000-2015." IFPRI publications.

Díaz-Bonilla, Eugenio. 2018. Financing "a sustainable food future": some thoughts for the G20. Economics Discussion Paper No. 2018-73. Kiel Institute for the World Economy. http://www.economics-ejournal.org/economics/discussionpapers/2018-73

Diaz-Bonilla, Eugenio; and Callaway, Victoria. 2018. The G20, global trade and investment regimes, and a sustainable food future. In How the G20 Can Help Sustainably Reshape the Global Trade System: A Compilation of Analysis. Part 3: Natural Environment. Pp 83-92. Geneva, Switzerland: International Centre for Trade and Sustainable Development (ICTSD). https://www.ictsd.org/themes/globaleconomic-governance/research/how-the-g20-can-help-sustainably-reshape-the-global-trade

Diaz-Bonilla, Eugenio; and Fernández-Arias, Eduardo. 2019. Políticas públicas y mercados financieros para el desarrollo rural y del sistema agroalimentario en América Latina y el Caribe. LAC Working Paper 2. Washington, DC: International Food Policy Research Institute (IFPRI). https://doi.org/10.2499/p15738coll2.133331

Diaz-Bonilla, E. y Saravia-Matus, S. 2019. Los objetivos de desarrollo sostenible y el desarrollo rural en américa latina y el caribe: reflexiones sobre costos y financiamientos. 2030 - Alimentación, agricultura y desarrollo rural en América Latina y el Caribe, No. 33. Santiago de Chile. FAO. 21 p.

FAO and GTZ. 1998. "Agricultural Finance Revisited" FAO y la Agencia Alemana de Cooperación Técnica (GTZ). 1998. Finanzas agrícolas revisadas: ¿por qué? FAO y GTZ Africa Series 1. Roma: FAO.

Fernández-Arias, E., R. Hausmann y U. Panizza. 2019. "Smart Development Banks". Journal of Industry, Competition and Trade (forthcoming). IADB manuscript available upon request.

Manfred Zeller and Richard Meyer eds 2002. The Triangle of Microfinance: Financial Sustainability, Outreach, and Impact. Johns Hopkins University.

Schmidt-Traub, G. y Sachs, J.D. 2015. Financing Sustainable Development: Implementing the SDGs through Effective Investment Strategies and Partnerships Working Paper. Revised ve rsion: 18 June 2015.

Seibel, HD, T. Giehler y S. Karduck. 2005. Reforma de los bancos de desarrollo agrícola. Munich: Agencia Alemana de Cooperación Técnica.

Funding for this work was provided in part by USAID. This publication has been prepared as an output of the ReSSAKS-LAC program and has not been independently peer reviewed. Any opinions expressed here belong to the author(s) and are not necessarily representative of or endorsed by IFPRI, IICA, IFAD, or USAID. 